\title{
Uso de Instagram para la comunicación de las marcas de moda de lujo
}

\author{
Marga Velar-Lera ${ }^{1}$ \\ Susana Herrera-Damas ${ }^{2}$ \\ Patricia González-Aldea ${ }^{3}$
}

Recibido: $31 / 07 / 2019$

Aprobado por pares: 23/08/2019
Enviado a pares: 02/08/2019

Aceptado: 03/09/2019

DOI: 10.5294/pacla.2020.23.4.4

Para citar este artículo / to reference this article / para citar este artigo Velar-Lera, M., Herrera-Damas, P. y González-Aldea, P. (2020). Uso de Instagram para la comunicación de las marcas de moda de lujo. Palabra Clave, 23(4), e2344. https://doi. org/10.5294/pacla.2020.23.4.4

\section{Resumen}

En un contexto de cambios globales, el propósito de esta investigación fue examinar de qué modo las marcas de moda de lujo pueden aprovechar este canal en su comunicación de marca y cómo lo están haciendo. Para ello, llevamos a cabo un análisis de contenido de las publicaciones realizadas por veinte marcas seleccionadas según criterios de antigüedad, relevancia y actividad digital. El periodo de estudio comprendía seis meses (julio-diciembre de 2017) para recoger la actividad en periodos de "normalidad" e intensidad. El corpus integrado por 6.977 publicaciones fue codificado a partir de un mismo código que incluía variables relativas al contenido y a cuestiones de tipo formal. Los resultados indican que las marcas de moda de lujo todavía no han diseñado estrategias específicas para Instagram. Advertimos que existe poca diferencia entre los contenidos que publican. La mayoría comparte un mismo patrón: presentan la marca en espacios similares (habitualmente en eventos o con fondos neutros), los personajes suelen encarnar uno o dos arquetipos y las imágenes suelen ser inexpresivas. Las marcas tampo- 
co aprovechan la capacidad que ofrece Instagram para estimular la participación y generar comunidad. Solo lo hace el 3,7\% del total de la muestra. De esta forma, concluimos que las marcas de moda de lujo en Instagram todavía no han logrado desarrollar una comunicación atractiva que genere interés y en la que realmente se busque construir la marca de un modo que se involucre a todos los públicos interesados.

\section{Palabras clave (Fuente: tesauro de la Unesco)}

Branding; marcas de moda de lujo; comunicación; Instagram; medios sociales; análisis de contenido. 


\section{Using Instagram for Communication of Luxury Fashion Brands}

\section{Abstract}

Amid global changes, this research intends to examine how luxury fashion brands can leverage this channel for brand communication and how they do it now. For this, we carried out a content analysis of the photos posted by 20 brands selected according to age, relevance, and digital activity criteria. The study period comprises six months (July-December 2017) to collect data during "normal" and intense periods. The integrating corpus of 6,977 posts was coded using the same code that included variables related to contents and formal questions. The results indicate that luxury fashion brands have not yet designed specific strategies for Instagram. We note little difference between the content they publish. Most share the same pattern: they exhibit the brand in similar spaces (usually at events or with neutral backgrounds), the characters often embody one or two archetypes, and images are often inexpressive. Brands do not take advantage of Instagram's ability to stimulate participation and create community neither; only $3.7 \%$ of the total sample do so. In brief, luxury fashion brands on Instagram have not yet developed attractive communication that holds followers' attention and actually builds the brand to engage the interested audiences.

\section{Keywords (Source: Unesco Thesaurus)}

Branding; fashion luxury brand; communication; Instagram; social media; content analysis. 


\section{Uso do Instagram para a comunicação das marcas de moda de luxo}

\section{Resumo}

Em um contexto de mudanças globais, esta pesquisa tem o objetivo de examinar de que forma as marcas de moda de luxo podem aproveitar o Instagram em sua comunicação de marca e como estão fazendo isso. Para isso, realizamos uma análise de conteúdo das publicações de 20 marcas selecionadas segundo critérios de antiguidade, relevância e atividade digital. $\mathrm{O}$ período de estudo foi de seis meses (de julho a dezembro de 2017) para coletar a atividade em períodos de "normalidade" e intensidade. O corpus esteve composto de 6.977 publicações e foi codificado a partir de um mesmo código que incluía variáveis relativas ao conteúdo e a questões de tipo formal. Os resultados indicam que as marcas de moda de luxo ainda não desenharam estratégias específicas para o Instagram. Observamos que há pouca diferença entre os conteúdos que postam. A maioria compartilha um mesmo padrão: apresentam a marca em espaços semelhantes (habitualmente em eventos ou com fundos neutros), as personagens costumam apresentar um ou dois arquétipos, e as imagens geralmente são inexpresivas. As marcas também não aproveitam a capacidade que o Instagram oferece para estimular a participação e gerar comunidade. Somente 3,7 \% da amostra faz isso. Dessa forma, concluímos que as marcas de moda de luxo no Instagram ainda não conseguiram desenvolver uma comunicação atrativa que gere interesse e na qual realmente se busque construir a marca de um modo que todos os públicos interessados estejam envolvidos.

\section{Palavras-chave (Fonte: tesauro da Unesco)}

Branding; marcas de moda de luxo; comunicação; Instagram; mídia social; análise de conteúdo. 


\section{Introducción}

Durante 2017, el mercado del lujo experimentó un crecimiento global del $5 \%$. Según el estudio desarrollado por Bain \& Co (D’Arpizio et al., 2017), las ventas de los objetos personales de lujo alcanzaron los EUR 262 billones, impulsadas por el resurgimiento de las compras de los clientes chinos tanto en su país como fuera, al igual que de otros grupos de clientes en otras regiones.

Un factor importante a la hora de entender este incremento es el escenario online. Según un estudio elaborado por la Fondazione Altagamma y Boston Consulting Group, el 72 \% de las compras de lujo realizadas por estadounidenses en 2016 se hicieron a través de internet. Para los clientes asiáticos y europeos, ese porcentaje fue del 64 y el $54 \%$, respectivamente (Ventura, 2017). Como se comprende, se trata de cifras significativas que convendría que las marcas consideraran al establecer sus estrategias.

Para Ian Rogers, chief digital officer (CDO) de LVMH, el principal reto de las marcas de moda de lujo es trasladar su universo a la experiencia online (Farled, 2017). Entre los nuevos canales, consideramos que Instagram es el que mejor responde a estas nuevas necesidades. Como afirma Alonso (2015, p. 84), se trata de la red visual más potente que existe y una herramienta de comunicación perfecta para la industria de la moda. Para algunos autores (Chu \& Kim, 2011; Erkan, 2015; Wallace et al., 2009), esta propiedad la convierte en la plataforma que mayor confianza y credibilidad genera en los usuarios, lo que se traduce en un mayor engagement. Su tasa se incrementa por encima de las de Twitter y Facebook (L2 Fashion Team, 2017; PMX Agency, 2017; Silva, 2014). Además, es en este canal en el que se encuentran los futuros clientes de las marcas de moda de lujo. Se estima que en 2026 la mayor parte de los clientes del lujo serán millennials. Actualmente, el $34 \%$ de los usuarios de Instagram pertenecen a esta generación (Statista, 2018).

Para lograr desarrollar una comunicación en Instagram que se oriente a construir marca, es necesario conocer bien las características del canal y producir contenidos que se ajusten a su singularidad. En caso contrario, 
Instagram quedaría convertido en un medio de comunicación unidireccional adicional en el que se adaptan los contenidos que se producen para medios tradicionales, sin procurar la interacción del receptor.

\section{Potencial de Instagram para la comunicación de las marcas de moda de lujo}

Como afirman algunos autores (Kim \& Ko, 2010; Okonkwo, 2009), inicialmente las marcas de moda de lujo no mostraron un gran interés en integrar estos nuevos canales, cuya naturaleza resulta aparentemente opuesta. El lujo procura mantener una imagen excepcional (Keller, 2009) y permanecer exclusivo para un segmento de mercado, mientras que los medios sociales tratan de llegar a un público muy amplio y defienden un contenido de libre acceso (Üçok et al., 2016).

Con todo, hoy la industria del lujo reconoce el papel tan importante que desempeñan internet, los medios y las redes sociales, "para mejorar la reputación de una marca, aumentar las interacciones con los clientes, y estimular sus deseos de lujo" (Ng, 2014, citado por Faraoni et al., 2016, p. 254).

Los medios sociales modifican las reglas del juego a la hora de llegar a nuevos consumidores y mercados. Sin embargo, como defienden algunos autores (Phan et al., 2011; Seo \& Buchanan-Oliver, 2015), la incorporación de estos medios también supone un cambio cultural, puesto que la marca deja de ser propiedad absoluta de la compañía al entrar a participar los usuarios y no como meros intermediarios. Es necesario que las marcas de moda de lujo tengan esto en cuenta, pues, según Phipps (2009), citado por Phan et al. (2011, p. 220), cuando las empresas reducen su actividad a captar personas influyentes, devalúan la confianza en la marca, ya que los consumidores perciben que están siendo utilizados.

En cambio, si se emplean de un modo adecuado, los medios sociales pueden ser un instrumento de comunicación valioso para potenciar el engagement, y así favorecer "una íntima relación con el cliente a largo plazo" (Sashi, 2012; The Economist Intelligence Unit, 2007). Para que esto ocurra, las marcas deben ir más allá y plantearse cómo crear y mantener una 
presencia digital "sin dañar su identidad de marca y el espíritu del lujo" (Maman et al., 2014, p. 198). Lograr este equilibrio puede ser uno de los mayores desafíos que afrontan las marcas de moda de lujo para su branding en social media (Üçok et al., 2016).

Los estudios académicos desarrollados en torno al luxury branding y los social media son todavía escasos (Alonso, 2015; Clavijo et al., 2017; Coursaris et al., 2013; Jin, 2012; Kim \& Ko, 2012; Gensler et al., 2013; Godey et al., 2016; Granitz \& Forman, 2015; Phan et al., 2011; Phua et al., 2017; Singh \& Sonnenburg, 2012). Los pocos que existen han centrado su atención de modo preferente, sobre todo, en Facebook y Twitter, si bien en los últimos años, esto ha comenzado a cambiar ante el auge de plataformas visuales como Instagram, YouTube o Snapchat (Kemp, 2018), y también ante la creciente incorporación de imágenes y vídeos en otras plataformas que, en sus orígenes, basaron su contenido, en especial, en contenido de tipo textual (Russmann \& Svensson, 2016, p. 1).

Instagram es uno de los medios que más ha crecido en los últimos años. A juicio de Moreno (2018, p. 229), se trata del "mayor caso de éxito de la última década en el universo de las redes sociales". Aunque su propósito original no ha cambiado mucho, ha ido incorporando algunas pequeñas mejoras y ajustes, como se puede apreciar en la figura $1^{4}$ (Fifteen, 2017; Instagram Press, 2017; Liebhart \& Bernhardt, 2017).

Desde su lanzamiento en octubre de 2010, Instagram ha logrado atraer a más de 1000 millones de usuarios en todo el mundo. Más de 500 millones de ellos se conectan a diario y el $80 \%$ siguen a alguna empresa en esta red (business.instagram.com, con datos obtenidos el 20 de enero de 2019) (figura 2).

Además, las características de este servicio le permiten ofrecer un gran potencial para la comunicación de las marcas de moda de lujo. En concreto (Velar, 2019):

4 La disponibilidad de las actualizaciones que ofrece Instagram varía en función del sistema operativo del dispositivo que se utilice y también según el país donde se encuentre el usuario. 
- Crea un espacio de marca que contextualiza la comunicación

- Permite escuchar y conocer al público

- Favorece la creación de comunidades en torno a la marca

- Ofrece una nueva forma de servicio personalizado

- Facilita la identificación de prescriptores

- Propicia la interacción de los usuarios con la marca

\section{Figura 1. Evolución funcional de Instagram (2010-2018).}

\section{0 \\ Instagram fue lanzado en el Apple App Store el 6 de octubre de 2010.}

\section{3}

- Introduce la posibilidad de etiquetar a personas y marcas en cualquiera de las fotos.

- Integra Instagram Direct, para enviar mensajes directos y privados con fotografías o videos.

\section{5}

- Las empresas ya pueden gestionar sus campañas a través de Facebook Business Manager

- Introduce las herramientas Layout y Boomerang.

\section{8}

- GIF stikers y nuevas actualizaciones.
2011

- Hashtags

- Versión 2.0 con nuevos filtros en vivo, opción de aplicar efectos de desenfoque a áreas concretas, edición de imágenes de alta resolución, bordes opcionales, botón de rotación de la imagen y un botón de actualización.

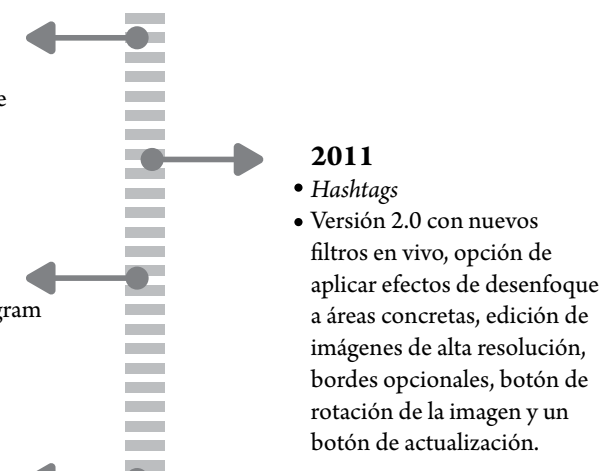

2014

- Introduce la aplicación para Instagram de Hyperlapse (solo para iPhone).

- Incorpora 5 nuevos filtros.
2016

- Instagram Stories y Zoom.

- Renueva su logotipo.

2017

- Live Stories, transmisión en directo.

- Etiquetado de personas y lugares en videos.

Fuente: elaboración propia según información publicada en Instagram Press. 
Figura 2. Evolución de Instagram por número de cuentas.

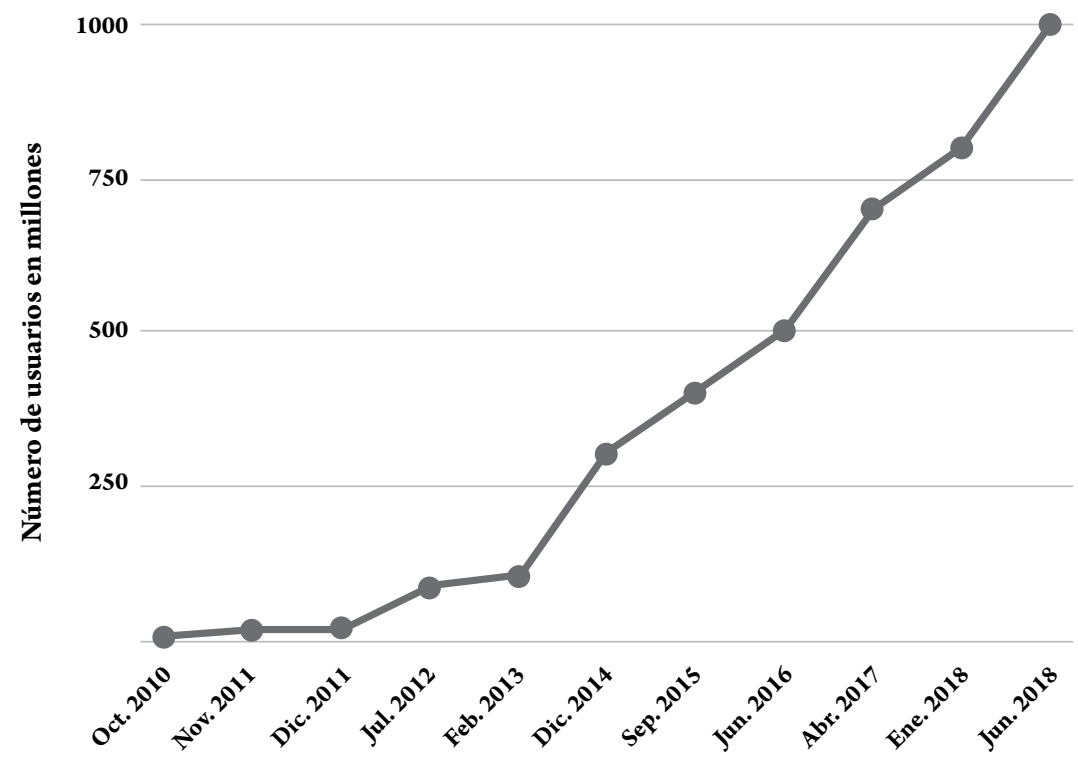

Fuente: elaboración propia según datos publicados en Instagram Press.

\section{Crea un espacio de marca que contextualiza la comunicación}

A diferencia de lo que ocurría habitualmente con la publicidad, en Instagram la marca dispone de un espacio donde recrear su universo y al que el usuario acude libremente (Villena, 2014, p. 18).

Además, las múltiples herramientas que ofrece Instagram facilitan tanto a la marca como al usuario publicar contenido creativo de calidad. Esta antigua relación del lujo y el arte cobra hoy una especial importancia. En el estudio elaborado por Escobar (2016, p. 28), se identificó entre los millennials a un grupo de usuarios activos en el sector de moda de lujo y con gran interés en su relación con la moda y el arte.

\section{Permite escuchar y conocer al público}

Según Roncha y Radclyffe-Thomas (2016, p. 306), Instagram está facilitando a las marcas conocer más profundamente a sus clientes actuales y llegar a los potenciales descubriendo sus motivaciones. A este proceso se le denomina social listeningy, según Chaffey \& Smith (2013, pp. 235-236), permite: 
- Comprender el mercado y a los clientes

- Evaluar las menciones que se hacen de la marca

- Identificar influencers

- Desarrollar relaciones de colaboración

- Encontrar ideas para crear contenido y desarrollar campañas

- Gestionar los comentarios negativos

- Identificar oportunidades de venta

- Desarrollar productos y servicios según las necesidades de los clientes

\section{Favorece la creación de comunidades en torno a la marca}

Los clientes esperan de las marcas que se alineen con sus pasiones y valores personales. Como afirma Moreno (2015, p. 24), el usuario no quiere recibir mensajes publicitarios con un marcado sesgo comercial, sino que desea sentirse parte de una comunidad.

Instagram es uno de los medios sociales que mayor engagement genera; por eso, resulta apropiado para crear comunidades en torno a las marcas (Moreno, 2018, p. 234).

Como recuerdan Mangold \& Faulds (2009, p. 361), el principal objetivo de la comunicación humana es conectar con personas con las que se comparten intereses. En este sentido, los medios sociales se convierten hoy en el canal idóneo, ya que, como afirman Roncha y Radclyffe-Thomas (2016, p. 305), permiten una mayor conectividad y más oportunidades para construir relaciones fuertes y duraderas.

\section{Ofrece una nueva forma de servicio personalizado}

Según un estudio elaborado por Deloitte (2017, p. 5) sobre el consumo de lujo en millennials, la tienda premium, con experiencias tangibles y contacto personal, está dando paso a un escenario menos estructurado y más complejo de administrar. Díaz \& García (2016, p. 148) afirman que, aunque las tiendas físicas suponen todavía el 70-80 \% de las ventas globales, han pasado 
a ser fundamentalmente espacios de experiencia. Las marcas de moda de lujo concentran sus esfuerzos en interactuar con sus clientes y conectar las tiendas tradicionales con los medios sociales. En este sentido, la decisión de Instagram de introducir una herramienta de venta es acertada y responde a la necesidad del cliente. Según Abraham (2011), el $41 \%$ de los millennials realizan sus compras a través del smartphone. Con esta función, el usuario puede seleccionar un producto que le gusta desde el post que está viendo, obtener más información e, incluso, comprarlo.

\section{Facilita la identificación de prescriptores de marca}

Los medios sociales han permitido democratizar el control de la información. En este sentido, el consumidor adquiere el poder de comentar en las redes su propia experiencia con marcas y productos.

Según Ortega y García (2014, p. 81), para los consumidores de lujo más jóvenes, la posibilidad de compartir en redes su experiencia con un determinado producto influye en su nivel de interés y relación con la marca. Esto ha favorecido que se desarrolle una verdadera red de prescripción a través de una técnica tan antigua como fiable: el boca-oreja (Chu \& Choi, 2011; Chu \& Kim, 2011). Este concepto tan estudiado en la década de los cincuenta sigue siendo una de las formas de comunicación más eficaces (Escobar, 2016, p. 25).

A juicio de Erkan (2015, p. 1437), cada vez resulta más frecuente que los consumidores acudan a los medios sociales para obtener información sobre las marcas (Baird \& Parasnis, 2011; Naylor et al., 2012), donde encuentran personas a las que perciben como cercanas.

Los clientes activos en redes se convierten en agentes clave para la marca, en embajadores, pues, como afirma Heath (2000, p. 81), se trata de personas con las que es fácil identificarse: "Las personas se identifican con aquellos en quienes confían. Confían en aquellos con quienes se identifican. Y confían en quienes representan y promueven narrativas que aceptan y adoptan" (citado por Kent, 2015, p. 481). 


\section{Propicia la interacción de los usuarios con la marca}

Según el informe “Trend report: Luxury brands online”, desarrollado por la PMX Agency (2017), el 7,4 \% del tráfico en la web proviene de los medios sociales de la propia marca. Como publica también L2 Fashion Team (2017) en su estudio sobre marcas de moda de lujo y medios sociales, el aumento del número de seguidores de estas cuentas fue del $53 \%$ en Instagram, del $28 \%$ en YouTube y del $11 \%$ en Facebook.

También fue Instagram la plataforma que registró una mayor participación. Al ser eminentemente visual, facilita que el usuario reaccione más rápido. Esto la convierte en una plataforma social clave para la industria del lujo con un ratio medio de engagement del 0,63 \% (PMX Agency, 2017, pp. 25 y 29$)$.

\section{Método}

En este contexto, el objetivo de este artículo consiste en analizar el uso real que las marcas de moda de lujo de referencia están haciendo de Instagram. Para llevar a cabo este estudio, hemos llevado a cabo un análisis del contenido de los mensajes publicados por veinte de ellas. Para diseñarlo, hemos consultado las aportaciones de Bardin (1986), Igartua (2006), Kerlinger (1973), Krippendorf (1990), Wimmer y Dominick (1996, 2006).

Revisadas sus contribuciones, seleccionamos las cuentas en Instagram de las principales marcas de moda de lujo de origen femenino, en atención a un conjunto de criterios:

- $\quad$ Antigüedad

- Participación en la alta costura

- Pertenencia a asociaciones de lujo

- $\quad$ Actividad en redes sociales

- Número de seguidores que tenía su cuenta en Instagram

En la tabla 1, presentamos las marcas seleccionadas, su fecha y lugar de nacimiento, y el número de seguidores que tenía su cuenta en Instagram en el momento en el que extrajimos los datos (abril de 2017). 


\section{Tabla 1. Tabla de marcas seleccionadas}

Marcas analizadas

\begin{tabular}{|c|c|c|c|c|c|}
\hline HERMES & $\begin{array}{c}\text { Francia } \\
1837\end{array}$ & $4,4 \mathrm{M}^{*}$ & CÉLINE & $\begin{array}{c}\text { Francia } \\
1945\end{array}$ & $86,3 \mathrm{k}$ \\
\hline LOEWE & $\begin{array}{c}\text { España } \\
1846\end{array}$ & $578 \mathrm{k}$ & or & $\begin{array}{c}\text { Francia } \\
1947\end{array}$ & $14,3 \mathrm{M}$ \\
\hline LOUIS VUITON & $\begin{array}{c}\text { Francia } \\
1854\end{array}$ & $15,3 \mathrm{M}$ & 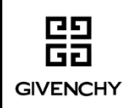 & $\begin{array}{c}\text { Francia } \\
1952\end{array}$ & $7,8 \mathrm{M}$ \\
\hline BURBE & $\begin{array}{c}\text { Reino } \\
\text { Unido } \\
1856\end{array}$ & $8,9 \mathrm{M}$ & Chloé & $\begin{array}{c}\text { Francia } \\
1952\end{array}$ & $4,1 \mathrm{M}$ \\
\hline LANVIN & $\begin{array}{c}\text { Francia } \\
1889\end{array}$ & $3,1 \mathrm{M}$ & VALE $\backslash T I N O$ & $\begin{array}{l}\text { Italia } \\
1960\end{array}$ & $8,4 \mathrm{M}$ \\
\hline & $\begin{array}{c}\text { Francia } \\
1909\end{array}$ & $18,8 \mathrm{M}$ & SAINT LAURENT & $\begin{array}{c}\text { Francia } \\
1961\end{array}$ & $1,7 \mathrm{M}$ \\
\hline PRADA & $\begin{array}{l}\text { Italia } \\
1913\end{array}$ & $11,5 \mathrm{M}$ & $\begin{array}{l}\text { BOTTEGA } \\
\text { VENETA }\end{array}$ & $\begin{array}{l}\text { Italia } \\
1966\end{array}$ & $824 k$ \\
\hline BALENCIAGA & $\begin{array}{c}\text { España } \\
1919\end{array}$ & $3,2 \mathrm{M}$ & ARMANI & $\begin{array}{l}\text { Italia } \\
1975\end{array}$ & $7,6 \mathrm{M}$ \\
\hline $\mathrm{G} \cup \mathrm{CCl}$ & $\begin{array}{l}\text { Italia } \\
1921\end{array}$ & $13,3 \mathrm{M}$ & $\begin{array}{l}\text { BRUNELLO } \\
\text { CUCINELLI }\end{array}$ & $\begin{array}{l}\text { Italia } \\
1978\end{array}$ & $157 \mathrm{k}$ \\
\hline FENDI & $\begin{array}{l}\text { Italia } \\
1925\end{array}$ & $6,9 \mathrm{M}$ & $\begin{array}{c}3 \\
\text { VERSACE }\end{array}$ & $\begin{array}{l}\text { Italia } \\
1978\end{array}$ & $8,6 \mathrm{M}$ \\
\hline
\end{tabular}

* Número de seguidores que tenía el perfil al comenzar el estudio (abril de 2017)

Fuente: elaboración propia.

La unidad de análisis que hemos escogido ha sido la publicación o el post entendiéndolo como el contenido que comparte en este caso cada marca, sin considerar las reacciones que suscite. ${ }^{5}$

El código que hemos diseñado para llevar a cabo el análisis incluye tanto aspectos formales - empleo de diferentes formatos, hashtags, etc.-

5 En este estudio, examinamos la comunicación desde el punto de vista del emisor, por lo que solo consideraremos el contenido que publique la marca. 
como cuestiones de fondo relacionadas con la finalidad del mensaje u otros elementos propios del contenido. El periodo de estudio comprende seis meses, entre julio y diciembre de 2017. El corpus quedó compuesto por 6977 publicaciones que fueron codificadas directamente por una de las autoras del artículo, tras una prueba piloto.

Tras los ajustes, comenzamos a codificar cada una de las 6977 publicaciones que componen la muestra de acuerdo con las definiciones establecidas. Lo hicimos primero en Excel. Después exportamos los datos al programa estadístico SPSS (versión 23.0) para extraer las frecuencias y tablas de contingencia, y conocer cómo se comporta cada una de las marcas. Para ello, definimos cada una de ellas como variable dependiente y la cruzamos después con el resto de las variables a las que tratamos como independientes.

\section{Resultados}

\section{Actividad en Instagram}

Como decimos, durante el periodo objeto de estudio, de julio a diciembre de 2017, las veinte marcas de moda de lujo seleccionadas realizaron 6977 publicaciones. Esto supone una media de 349 publicaciones por marca y de casi dos diarias $(1,9)$ por cada una de ellas. No obstante, el análisis por marcas muestra una realidad muy diferente.

En los 184 días que integraron el periodo objeto de estudio, la marca con mayor número de publicaciones fue Gucci, con 675 y una media diaria de 3,7 posts al día. Su actividad difiere mucho de los 15 posts en total que publica Brunello Cucinelli, la marca con menor actividad. Lanvin es la que más se ajusta a la media con 334 publicaciones y una media de 1,8 por día. Los resultados para cada una de las marcas quedan reflejados en la figura 3.

Respecto del uso de formatos, las más innovadoras fueron Saint Laurent, Prada, Chanel y Versace, marcas que hicieron un uso equilibrado de todos ellos (tabla 2). 
Figura 3. Número de publicaciones por marca.

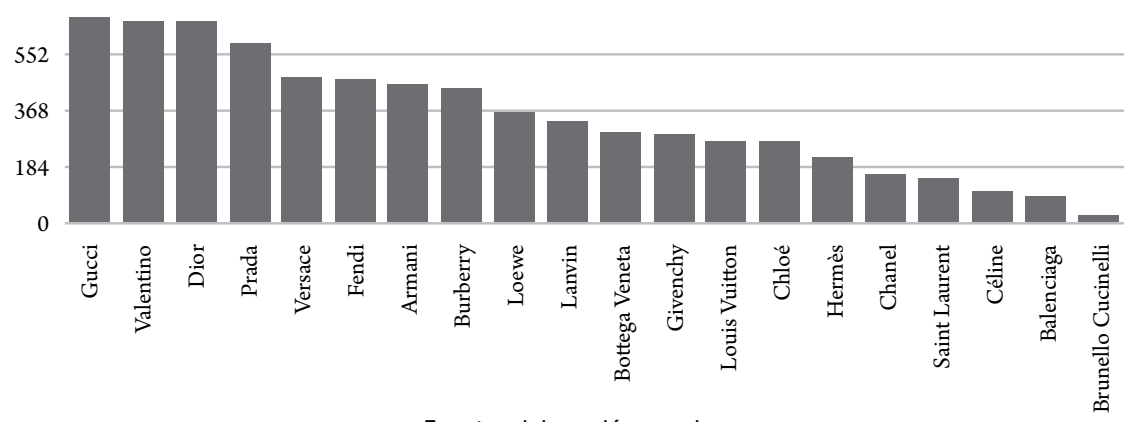

Fuente: elaboración propia.

Tabla 2. Marcas más innovadoras en el uso de tipos de post

\begin{tabular}{|c|c|c|c|c|}
\hline & Saint Laurent & Prada & Chanel & Versace \\
\hline Imagen & $57,6 \%$ & $57,9 \%$ & $54,7 \%$ & $77,9 \%$ \\
\hline Video & $25,7 \%$ & $23 \%$ & $33,3 \%$ & $14 \%$ \\
\hline Puzle & $8,3 \%$ & $12,7 \%$ & $3,8 \%$ & $3,8 \%$ \\
\hline Composición & $8,3 \%$ & $6,4 \%$ & $8,2 \%$ & $4,4 \%$ \\
\hline
\end{tabular}

Fuente: elaboración propia.

\section{Uso de los recursos que ofrece Instagram}

El hashtag o etiqueta es un recurso habitual en las publicaciones de las marcas analizadas. Como se observa en la figura 4, lo más común es utilizarlos para etiquetar, sobre todo, colecciones y, en menor medida, campañas o eventos.

Figura 4. Frecuencia de uso de hashtags para el total de la muestra.

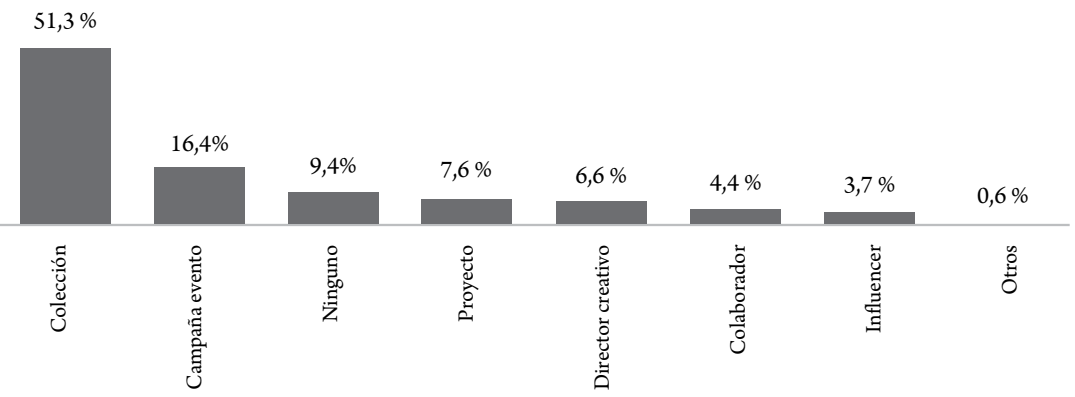

Fuente: elaboración propia. 
Esta estrategia tiene sentido si pensamos que las etiquetas sirven para catalogar las publicaciones por temas. Este tipo de títulos facilitarán que los usuarios encuentren la marca cuando busquen las novedades de la temporada: \#FW18, por ejemplo, hace referencia a la colección de otoño-invierno 2018 (Fall-Winter). En \#EmmyAwards, se pueden consultar los vestidos que llevaron los famosos a la gala de entrega de los premios Emmy.

A diferencia de lo que ocurría con los hashtags, las menciones se suelen utilizar menos: el $46 \%$ de las publicaciones analizadas no incluyen ninguna en sus publicaciones. Además, cuando lo hacen, suele ser preferentemente a personas particulares y, en especial, a aquellas con las que la marca haya llevado a cabo alguna colaboración (16\%).

En cuanto al uso de enlaces, los resultados muestran que las marcas apenas los incluyen en sus publicaciones. Del total de mensajes analizados, solo el $31 \%$ deriva al usuario hacia sitios externos, habitualmente a la propia web a través del recurrente \#linkinbio (15\%) o recordando la dirección de la web (12\%).

\section{Finalidad de los mensajes}

Si analizamos el propósito de los mensajes, advertimos que los más habituales son dar a conocer un producto (48\%) o subrayar la notoriedad de marca $(38,2 \%)$. Por marcas, la distribución de estas finalidades resulta bastante similar. En más de la mitad, predomina el objetivo de dar a conocer un producto: en Lanvin, encontramos esta finalidad en el $77 \%$ de sus publicaciones, Versace (63\%), Chanel (60\%), Fendi (58\%), Hermès (57\%), Armani (55\%), Céline (55\%), Gucci (53\%), Bottega Veneta $(53 \%)$, Balenciaga (48\%), Prada (48\%), Dior (46\%), Loewe (44\%) y Louis Vuitton (37\%).

En las seis marcas restantes, la principal intención es subrayar la notoriedad de la marca. Así ocurre en el $73 \%$ de la muestra de Brunello Cucine1li, Saint Laurent (59\%), Burberry (59\%), Chloé (51\%), Valentino (46\%) y Givenchy (44\%). En la figura 5, Burberry apoya la estrategia de algunos de sus productos icónicos: el trench y el estampado de cuadros (figura 5). 


\section{Figura 5. Ejemplo de publicación que subraya}

la notoriedad de la marca.

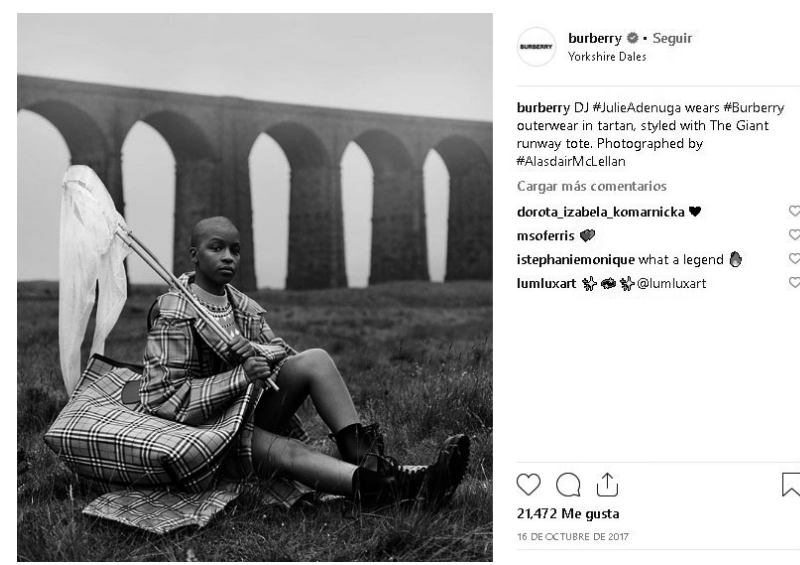

Fuente: Instagram.

\section{Presencia de la marca}

Los elementos gráficos de la marca son símbolos clave para su identificación y posterior notoriedad. En Instagram, el logo se ha convertido en una señal útil para que las marcas puedan diferenciar sus imágenes de las de la competencia en un canal saturado de ellas. Los resultados muestran que el logo aparece en un 43,6 \% del total de publicaciones.

No obstante, en el análisis por marcas, la distribución es muy desigual. Entre las que más utilizan el logo, están Louis Vuitton (69 \%) y Brunello Cucinelli (67\%). En el extremo opuesto, con bastante diferencia entre sí, se encuentran Céline ( $8 \%)$, Armani (18\%) y Givenchy (22\%).

Otros elementos útiles que permiten reconocer la marca y que pueden servir de soporte para construir su identidad son el producto, el arte, el estilo comunicativo, el origen, el fundador/director creativo o la artesanía. Sin embargo, como podemos ver en la tabla 3, el recurso a estos elementos todavía resulta escaso.

Por marcas, las que hacen un mayor uso del producto como soporte narrativo son Hermès (56\%), Loewe (45\%) y Valentino (33 \%), que cuentan con productos icónicos o elementos reconocibles. 
Tabla 3. Elementos narrativos más empleados por las marcas

\begin{tabular}{|c|c|c|}
\hline Elemento de marca & $\begin{array}{c}\mathbf{N .}^{\circ} \text { de publicaciones sobre el total de } \\
\text { la muestra }\end{array}$ & \% sobre el total de publicaciones \\
\hline Producto & 947 & $13,6 \%$ \\
\hline Arte/cultura & 773 & $11,1 \%$ \\
\hline Estilo comunicativo & 258 & $3,7 \%$ \\
\hline Origen & 238 & $3,4 \%$ \\
\hline Fundador/director creativo & 188 & $2,7 \%$ \\
\hline Artesanía & 148 & $2,1 \%$ \\
\hline
\end{tabular}

Fuente: elaboración propia.

Respecto del uso de otros elementos, destaca Brunello Cucinelli que se apoya en partes iguales en la figura de su fundador (33\%) y en su origen (33\%). También lo hace Saint Laurent en su estilo comunicativo (32\%) caracterizado por el blanco y el negro, y la sofisticación de figuras marginales. En la comunicación de Gucci, están muy presentes el arte y los referentes culturales, que encontramos en un $37 \%$ de su muestra.

El espacio donde se desarrolla la comunicación de las marcas es parte del mensaje que se quiere transmitir. De los datos del análisis, se desprende que los espacios por excelencia son la pasarela o el evento (25\%) y los neutros (23\%). Estos últimos son espacios limpios, sin referentes, donde el producto o el modelo atrae toda la atención y genera una sensación de atemporalidad.

Junto con el espacio, el sonido que emplea la marca en sus publicaciones en vídeo también es otro indicador del ambiente con el que se identifica. Los resultados reflejan que, entre los posts que contienen audio, el sonido más común es la música moderna. Así ocurre en el $51 \%$ de las publicaciones.

\section{Relación de las marcas con sus usuarios}

Tradicionalmente, este tipo de marcas han utilizado los primeros planos para reforzar así el protagonismo del producto (Sicard, 2008). El análisis realizado refleja que en Instagram sigue ocurriendo lo mismo: el $43 \%$ de las publicaciones son imágenes en primer plano. El resto de las publicaciones se reparten, sobre todo, entre los planos enteros $(28 \%)$ y el cinematográ- 
fico, llamado también plano americano (19\%). Los planos de detalle son muy escasos y solo los encontramos en el $9 \%$ del total de la muestra. Los planos abiertos de paisaje no son estadísticamente significativos.

El tipo de ángulo indica en cierto modo qué clase de relación quiere establecer la marca con su público. Los resultados del estudio reflejan un cambio de posición respecto de las imágenes tradicionales con planos tomados desde el ángulo contrapicado. La mayoría de las publicaciones emplean un ángulo frontal (78 \%), lo que genera una relación más cercana con los usuarios.

Existe un tipo de ángulo más residual que es el cenital. Lo utilizan, aunque en menor medida, marcas como Dior (7\%) y Chanel (5\%) para mostrar parte de sus procesos creativos.

El servicio personalizado ha sido siempre una nota característica del lujo. Por eso, nos parecía interesante analizar cómo actúan en este sentido las marcas de moda de lujo en Instagram. El servicio solo está presente en el $7 \%$ del conjunto de publicaciones. Sin embargo, en el análisis por marcas, observamos que en Louis Vuitton este tema ocupa el $20 \%$ de su muestra.

A diferencia del servicio, la llamada a la participación es una característica propia de nuestro tiempo, de una sociedad y un mercado conscientes de la importancia de todos los públicos implicados en la actividad de una compañía. Sin embargo, los datos revelan un comportamiento que se aleja de esta práctica. Solo encontramos esta finalidad en un $4 \%$ del total de la muestra y tampoco tiene un gran peso en ninguna de las marcas.

En general, el principal objetivo de la comunicación en lujo no es tanto fomentar la compra de productos, sino crear universos en los que el cliente se sumerja. Por eso, no es habitual encontrar entre las marcas anuncios de promociones o descuentos. Sin embargo, esto, que hasta ahora ha sido un precepto tradicional, parece que no se cumple del todo en la comunicación digital, ya que casi un $5 \%$ del total de las publicaciones incluyen un llamamiento explícito a la compra. No obstante, esta práctica no pre- 
senta una distribución homogénea en el conjunto de la muestra, ya que la encontramos solo en algunas marcas como Versace (34\%), Chloé (14\%), Burberry (14\%) y Loewe (6\%).

\section{Personajes con los que asocia la marca}

Aunque el fundador o el director creativo suelen tener un papel casi determinante en la construcción de la personalidad de la marca, solo aparece en el $7 \%$ de las publicaciones analizadas. Así ocurre, sobre todo, en el $33,3 \%$ de las publicaciones de Brunello Cucinelli, Chanel (11,9\%) y Versace $(10,2 \%)$.

En el primer caso, la influencia de Cucinelli es fuerte y la filosofía de la marca se basa en el pensamiento humanista de su creador que resume en una frase del escritor ruso Fiodor Dostoyevski: "La belleza salvará al mundo”. En Chanel, gran parte de su comunicación sigue pivotando en torno a la figura de Gabrielle Chanel (1883-1971). Su director creativo, siendo un genio, es capaz de devolver a la vida a su fundadora. El fundador de Versace, creada en el mismo año que Brunello Cucinelli, Gianni, murió asesinado en 1997. Pese a ello, su persona sigue estando presente en la marca gracias a la dirección de su hermana Donatella que se quedó al mando de la firma.

Además de la influencia que pueda ejercer el fundador o director creativo, la personalidad de la marca también se construye a través de los arquetipos que encarna. En este análisis, hemos utilizado un indicador simplificado, basándonos en la propuesta de Aaker (1997). Este autor presenta solo cinco rasgos de personalidad. Aunque las limitaciones de espacio no nos permiten examinar los personajes de un modo más profundo, advertimos que el arquetipo más representado en el total de la muestra es el soñador, seguido del sofisticado. Se trata de una relación lógica si consideramos que una de las finalidades de las marcas de moda de lujo es hacer sonar. Los resultados se pueden apreciar en la figura 6.

Otra variable que permite analizar la personalidad de la marca es la expresión personal o el gesto que presentan. Los datos reflejan que, en la mayoría de las imágenes en las que aparece alguna persona, esta se muestra inexpre- 


\section{Figura 6. Arquetipos más representados en el conjunto}

de la muestra.

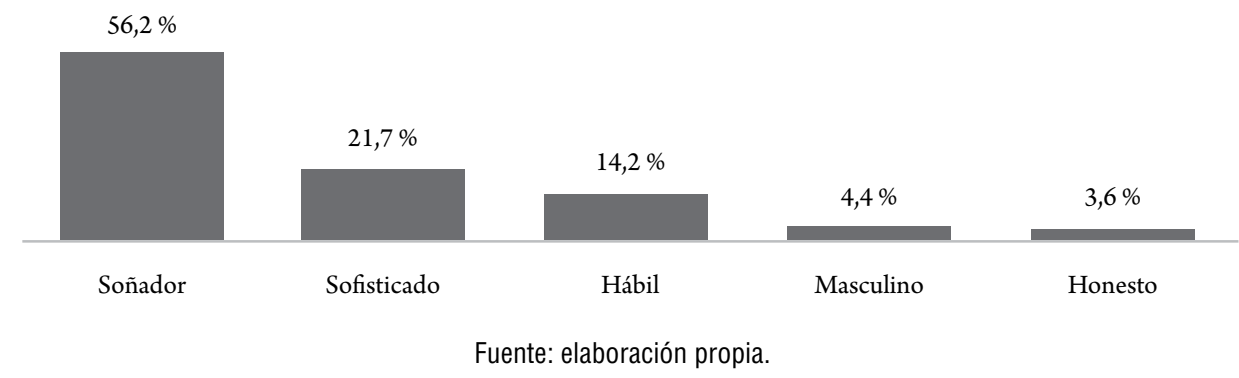

siva $(44 \%)$. Esta falta de expresividad tiene sus orígenes en la Inglaterra del siglo XVII, cuya aristocracia nunca mostraba sus sentimientos o emociones (Jeeves, 2013). Hoy día, en las pasarelas, seguimos viendo esa inexpresividad en los rostros impertérritos de los modelos que desfilan. Por marcas, impera la falta de expresión en seis de las veinte marcas que hemos analizado: Lanvin (78 \%), Balenciaga (70\%), Givenchy (55\%), Loewe (54\%), Bottega Veneta (52\%) y Brunello Cucinelli (50\%).

Entre las imágenes que reflejan cierta expresividad, lo más habitual es que predomine la amabilidad (23\%) y el disfrute (20\%). También encontramos publicaciones en las que sus protagonistas adoptan un gesto seductor $(8 \%)$ o de reto $(5 \%)$.

Los influencers o personajes con los que se asocia la marca también son una variable interesante a la hora de analizar su personalidad, ya que permiten ver con quién se identifica o quiere identificar. Las marcas que más veces publicaron imágenes con influencers son Saint Laurent (68 \%), Burberry (51\%), Chanel (50\%) y Bottega Veneta (47\%), aunque, en general, observamos que no se trata de una práctica muy extendida.

Los influencers con los que más se asocian las marcas son los que pertenecen a la cultura pop ( $52 \%$ ) y los modelos (35\%). Por marcas, y siempre en atención al total de posts en los que aparecen influencers, predominan los de cultura pop, excepto Loewe (21\%), Fendi (26\%), Versace (32\%), Saint Laurent (32\%) y Bottega Veneta (39\%). 


\section{Conclusiones}

Los resultados reflejan que la comunicación de las marcas de moda de lujo en Instagram resulta ambivalente y deja luces y sombras. Entre los aspectos más positivos destacamos:

1. El empleo coherente que hacen algunas marcas de sus logos y otros elementos estéticos con los que logran transmitir visualmente su identidad. En el 43,6 \% de las publicaciones que hemos analizado, resulta fácil reconocer la marca. Destacan Louis Vuitton, con un uso coherente del código visual en el 68,8 \%, Brunello Cucinelli (67\%), Chanel (59\%) y Gucci (59\%).

2. El uso actual que algunas marcas hacen del plano, lo que permite generar relaciones más cercanas. El $43 \%$ de las imágenes son primeros planos y en el $78 \%$ predomina el ángulo frontal.

3. La relación que algunas marcas de moda de lujo establecen con el arte $y$ con otras manifestaciones culturales permite enriquecer el propio relato. Entre ellas predomina Gucci, para la que el arte es un elemento narrativo, como se refleja en el $37 \%$ de sus publicaciones. Asimismo, el $22 \%$ de su contenido se presenta en un espacio artístico-cultural.

4. El acierto en la elección de influencers por parte de las marcas. Por ejemplo, Loewe, una de las más antiguas y a la vez más contemporánea desde la entrada de Jonathan Anderson, escoge en un $21 \%$ a influencers que pertenecen a la alta cultura, mientras que en otro $48 \%$ elige a personas que se han hecho famosas a través de las redes sociales.

Junto a estos indicadores, también encontramos otros más negativos. Algunos de los más disfuncionales son:

1. Las limitadas referencias al patrimonio de la marca tanto en relación con sus orígenes como con los procesos artesanales. Solo el 3,4 \% de los posts que hemos analizado presentan un contenido histórico y el $2,7 \%$ aluden a su fundador. Del mismo modo, únicamente el $2 \%$ del contenido narrativo de marca se apoya en el valor del trabajo artesanal. 
2. El escaso interés en generar comunidad en torno a la marca. Del total de la muestra, solo el $4 \%$ de las publicaciones se dirigen a fomentar la participación de los usuarios.

3. La falta de estrategia en el uso de hashtags. La mayoría se utiliza para etiquetar colecciones ( $51 \%$ ) o alguna campaña (16\%). Únicamente el $8 \%$ de la muestra etiqueta proyectos concretos.

4. La poca implicación de las marcas de moda de lujo en las cuestiones actuales y en los problemas sociales. Del total de publicaciones que hemos analizado, solo el 3 y el 0,5 \% tratan estos temas, respectivamente. Destaca, no obstante, la actuación de marcas como Louis Vuitton, Givenchy, Gucci o Dior, que recurren a esta práctica de un modo más habitual.

En general, estas marcas siguen un mismo patrón que se basa en adaptar contenidos tradicionales a medios online. En este escenario, resulta difícil que alguna marca sobresalga. La irrupción de nuevos canales como Instagram exige también un nuevo modo de diseñar los contenidos que responda a su singularidad.

En lugar de mostrarse auténticas y consistentes a través de una cuidada producción y selección de contenidos (Pahl et al., 2011), las marcas emplearon formas de comunicar muy similares. Esta falta de aprovechamiento del potencial que ofrece Instagram no solo se refleja en el tipo de contenido que se publica, sino que se extiende también al modo en que las marcas emplean otros recursos para llamar la atención de sus públicos, involucrarlos y crear comunidad.

De esta forma, podemos concluir que las marcas de moda de lujo en Instagram todavía no han logrado desarrollar una comunicación atractiva que genere interés y en la que realmente se busque construir la marca de un modo que se involucre a todos los públicos interesados.

De un modo más concreto, observamos que la comunicación de las marcas de moda de lujo en Instagram modifica algunos de los cánones clá- 
sicos que habían funcionado hasta ahora. A partir de ahí registramos una serie de similitudes y diferencias entre las marcas.

Si tuviéramos en cuenta solo los recursos que ofrece Instagram para innovar en el modo de involucrar a los usuarios, concluiríamos que las marcas más innovadoras son, en este orden, Balenciaga, Loewe, Valentino, Fendi, Givenchy, Chloé, Lanvin y Prada. Sin embargo, a la hora de analizar el comportamiento de las marcas seleccionadas, no hemos considerado solo los factores externos, sino también el origen y la evolución de cada marca.

En atención a estos datos, observamos las siguientes características. Algunas marcas han aprovechado las circunstancias para crear un nuevo estilo comunicativo. Hermès, Gucci, Prada y Burberry lo han hecho incorporando elementos de su herencia y aportando una gran riqueza al relato. De este grupo, todas ellas coinciden en el uso habitual de escenarios naturales en sus publicaciones. Este tipo de espacio rompe con la dinámica tradicional del lujo cargada de artificio y se identifica con el gusto actual hacia lo natural. Balenciaga, en cambio, ha emprendido un camino aparentemente ex novo y desde la incorporación a la firma del diseñador Demna Gvasalia su estética ha ido adoptando elementos underground muy alejados del diseño arquitectónico de su fundador, el modisto español Cristóbal Balenciaga.

En general, las marcas que más acciones desarrollan para fomentar la participación son las mismas que en alguna de sus publicaciones se refieren a la venta de una manera explícita. Entre ellas se encuentran marcas con una identidad diluida o una estrategia muy cercana a la de las marcas premium, lo que supone un riesgo de branding a largo plazo. En otras, este comportamiento se equilibra con otro tipo de mensajes de servicio o contenido relacionados con la cultura y el arte. Estas últimas suelen ser marcas sólidas, por lo que se pueden permitir publicar este tipo de mensajes sin caer en el comercialismo.

Los resultados revelan también que Instagram es una plataforma eficaz para el desarrollo de las relaciones de las marcas de moda de lujo cuando se considera el tipo de marca con la que se trabaja, su origen y la identidad 
que se pretende construir. No hay que olvidar que se trata de una herramienta, por lo que resulta necesario saber cómo utilizarla para sacar el mayor provecho.

Por lo demás, el hecho de haber basado en el análisis las publicaciones realizadas por las marcas constituye alguna limitación. Para valorar de un modo más completo el tipo de relación que las marcas de moda de lujo mantienen con sus usuarios, sería interesante analizar las reacciones de estos últimos a las publicaciones, stories y directos que realiza la marca. Además de la conversación que se desarrolla en el medio controlado por la marca, existe una gran actividad relacionada con ella en las cuentas de los propios usuarios y que es posible seguir a través de hashtags.

\section{Referencias}

Aaker, J. L. (1997). Dimensions of brand personality. Journal of Marketing Research, 34(3), 347-356. https://journals.sagepub.com/doi/ abs/10.1177/002224379703400304

Abraham, A. (2011). By the numbers: 50 facts about millennials. Edelman.

Alonso González, M. (2015). Las redes sociales como canal de comunicación de las marcas de moda españolas: el caso de Zara, Mango y el Corte Inglés. Index.comunicación: Revista Científica en el Ámbito de la Comunicación Aplicada, 5(1), 77-105. https://dialnet.unirioja.es/servlet/articulo? codigo $=5277295$

Baird, C. \& Parasnis, G. (2011). From social media to social customer relationship management. Strategy \& Leadership, 39(5), 30-37. https://doi.org/10.1108/10878571111161507

Bardin, L. (1986). Análisis de contenido. Akal.

Chaffey, D. \& Smith, P.R. (2013). eMarketing eXcellence: Planning and optimizing your digital marketing. Routledge. 
Chu, S. C. \& Choi, S. M. (2011). Electronic word-of-mouth in social networking sites: A cross-cultural study of the United States and China. Journal of Global Marketing, 24(3), 263-281. https://doi.org/ $10.1080 / 08911762.2011 .592461$

Chu, S. C. \& Kim, Y. (2011). Determinants of consumer engagement in electronic word-of-mouth (eWOM) in social networking sites. International Journal of Advertising, 30(1), 47-75. https://doi. org/10.2501/IJA-30-1-047-075

Clavijo Ferreira, L., Pérez Curiel, C. y Luque Ortiz, S. (2017). Social media y comunicación corporativa : nuevo reto en las empresas de moda. En Del verbo al bit. (pp. 2029-2059). Sociedad Latina de Comunicación Social. https://idus.us.es/handle/11441/61444

Coursaris, C. K., Van Osch, W. \& Balogh, B. A. (2013). A social media marketing typology: Classifying brand Facebook page messages for strategic consumer engagement. Proceedings of the 21st European Conference on Information Systems, 46, 46-58. https://aisel.aisnet. org $/ \mathrm{cgi} /$ viewcontent.cgi $?$ article $=1269 \&$ context $=$ ecis 2013 cr

D’Arpizio, C., Levato, F., Zito, D. \& De Montgolfier, J. (2017). Luxury goods worldwide market study, Fall-Winter 2017. Bain \& Company. https://www.bain.com/insights/luxury-goods-worldwidemarket-study-fall-winter-2017/

Deloitte. (2017). Bling it on: What makes a millennial spend more? https:// www2.deloitte.com/content/dam/Deloitte/uk/Documents/ consumer-business/deloitte-uk-young-luxury-shopper-2017.pdf

Díaz Soloaga, P. \& García Guerrero, L. (2016). Fashion films as a new communication format to build fashion brands. Communication \& Society, 29(2), 45-61. https://eprints.ucm.es/38526/

Erkan, I. (2015). Electronic word of mouth on Instagram: Customers' engagements with brands in different sectors. International Jour- 
nal of Management, Accounting and Economics, 2(12), 1435-1444. https://www.researchgate.net/profile/Ismail_Erkan/publication/292991785_Electronic_Word_of_Mouth_on_Instagram Customers'_Engagements_with_Brands_in_Different_Sectors/ links/56b4b40c08aec41daa206223.pdf

Escobar Ríos, A. (2016). The impact of the digital revolution in the development of market and communication strategies for the luxury sector (fashion luxury). Central European Business Review, 5(2), 17-36. https://doi.org/10.18267/j.cebr.149

Faraoni, M., Bandinelli, R. \& Rinaldi, R. (2016). Social media strategy in the italian fashion industry: A new model of analysis. En R. Rinaldi \& R. Bandinelli (Ed.), Workshop on Business Models and ICT Technologies for the Fashion Supply Chain. (pp. 253-269). Springer. https://doi.org/10.1007/978-3-319-48511-9_21

Farled, F. (2017, septiembre 21). Cómo comprar productos de lujo a través de chats. La Vanguardia. http://www.lavanguardia.com/de-mo$\mathrm{da} / \mathrm{moda} / 20170920 / 431422714589 /$ moda-online-lujo-redessociales-altagama.html

Fifteen. (2017, marzo 14). The evolution of Instagram - from camera app to the second most important social network. https://www.fifteendesign. co.uk/blog/the-evolution-of-instagram-from-camera-app-to-thesecond-most-important-social-network/

Gensler, S., Völckner, F., Liu-Thompkins, Y. \& Wiertz, C. (2013). Managing brands in the social media environment. Journal of Interactive Marketing, 27(4), 242-256. https://doi.org/10.1016/j. intmar.2013.09.004

Godey, B., Manthiou, A., Pederzoli, D., Rokka, J., Aiello, G., Donvito, R. \& Singh, R. (2016). Social media marketing efforts of luxury brands: Influence on brand equity and consumer behavior. Journal of Business Research, 69(12), 5833-5841. https://doi.org/10.1016/j. jbusres.2016.04.181 
Granitz, N. \& Forman, H. (2015). Building self-brand connections: Exploring brand stories through a transmedia perspective. Journal of Brand Management, 22(1), 38-59. https://doi.org/10.1057/ bm.2015.1

Heath, R. L. (2000). A rhetorical perspective on the values of public relations: Crossroads and pathways toward concurrence. Journal of Public Relations Research, 12(1), 69-91. https://doi.org/10.1207/ S1532754XJPRR1201_5

Igartua Perosanz, J. J. (2006). Métodos cuantitativos de investigación en comunicación. Bosch.

Instagram business. https://business.instagram.com/

Instagram Press. https://instagram-press.com/

Jeeves, N. (2013, septiembre 18). The serious and the smirk: The smile in portraiture. The Public Domain Review. https://publicdomainreview.org/2013/09/18/the-serious-and-the-smirk-the-smile-inportraiture/

Jin, S. A. (2012). The potential of social media for luxury brand management. Marketing Intelligence \& Planning, 30(7), 687-699. https:// doi.org/10.1108/02634501211273805

Keller, K. L. (2009). Managing the growth tradeoff: Challenges and opportunities in luxury branding. Journal of Brand Management, 16(56), 290-301. https://doi.org/10.1057/bm.2008.47

Kemp, S. (2018). Global digital report 2018. https://digitalreport.wearesocial.com/

Kent, M. L. (2015). The power of storytelling in public relations: Introducing the 20 master plots. Public Relations Review, 41(4), 480-489. https://doi.org/10.1016/j.pubrev.2015.05.011 
Kerlinger, F. N. (1973). Foundations of behavioral research. Holt, Rinehart and Winston.

Kim, A. J. \& Ko, E. (2010). Impacts of luxury fashion brand's social media marketing on customer relationship and purchase intention. Journal of Global Fashion Marketing, 1(3), 164-171. https:// doi.org/1 $0.1080 / 20932685.2010 .10593068$

Krippendorff, K. (1990). Metodología de análisis de contenido: teoría y práctica. Paidós.

L2 Fashion Team. (2017, noviembre 2). L2 Digital IQ Index: Fashion 2017. Autor.

Liebhart, K. \& Bernhardt, P. (2017). Political storytelling on Instagram: Key aspects of Alexander Van der Bellen's successful 2016 presidential election campaign. Media and Communication, 5(4), 1525. https://doi.org/10.17645/mac.v5i4.1062

Maman Larraufie, A. F. \& Kourdoughli, A. (2014). The e-semiotics of luxury. Journal of Global Fashion Marketing, 5(3), 197-208. https:// doi.org/10.1080/20932685.2014.906120

Mangold, W. G. \& Faulds, D. J. (2009). Social media: The new hybrid element of the promotion mix. Business Horizons, 52(4), 357-365. https://doi.org/10.1016/j.bushor.2009.03.002

Moreno, M. (2015). Cómo triunfar en las redes sociales. Gestión 2000.

Moreno, M. (2018). La enciclopedia del community manager. Deusto.

Naylor, R. W., Lamberton, C. P. \& West, P. M. (2012). Beyond the "like" button: The impact of mere virtual presence on brand evaluations and purchase intentions in social media settings. Journal of Marketing, 76(6), 105-120. https://doi.org/10.1509/jm.11.0105 
Okonkwo, U. (2009). The luxury brand strategy challenge. Journal of Brand Management, 16(5-6), 287-289. https://doi.org/10.1057/ bm.2008.53

Ortega, S. y García Hiljding, I. (2014). El sector del lujo en el entorno digital. En T. Sádaba (Ed.), La moda en el entorno digital. (pp. 7992). Eunsa.

PMX Agency. (2017). Trend report: Luxury brands online. https:// www.pmxagency.com/wp-content/uploads/2018/06/PMX_ LUXE2017_9-13-17_digital_nolv.pdf

Phan, M., Thomas, R. \& Heine, K. (2011). Social media and luxury brand management: The case of Burberry. Journal of Global Fashion Marketing, 2(4), 213-222. https://doi.org/10.1080/20932685.2011 .10593099

Phua, J., Jin, S. V. \& Kim, J.J. (2017). Gratifications of using Facebook, Twitter, Instagram, or Snapchat to follow brands: The moderating effect of social comparison, trust, tie strength, and network homophily on brand identification, brand engagement, brand commitment, and membership intention. Telematics and Informatics, 34(1), 412424. https://doi.org/10.1016/j.tele.2016.06.004

Roncha, A. \& Radclyffe-Thomas, N. (2016). How TOMS 'one day without shoes' campaign brings stakeholders together and co-creates value for the brand using Instagram as a platform. Journal of Fashion Marketing and Management, 20(3),300-321. https://doi.org/10.1108/ JFMM-10-2015-0082

Russmann, U. \& Svensson, J. (2016). Studying organizations on Instagram. Information, 7(4). https://doi.org/10.3390/info7040058

Sánchez Aranda, J.J. (2005). Análisis de contenido cuantitativo de medios. En M. R. Berganza y J. A. Ruiz San Román (Coords.), Investigar 
en comunicación: guía práctica de métodos y técnicas de investigación social en comunicación. (pp. 207-228). McGraw-Hill.

Sashi, C.M. (2012). Customer engagement, buyer-seller relationships, and social media. Management Decision, 50(2), 253-272. https://doi. org/10.1108/00251741211203551

Seo, Y. \& Buchanan-Oliver, M. (2015). Luxury branding: The industry, trends, and future conceptualizations. Asia Pacific Journal of Marketing and Logistics, 27(1), 82-98. https://doi.org/10.1108/ APJML-10-2014-0148

Sicard, M.-C. (2008). Lujo, mentiras y marketing: cómo funcionan las marcas de lujo. Gustavo Gili.

Silva Robles, C. (2014). Instagram, generación de contenidos y marcas de lujo: la narración de historias como estrategias de comunicación. En D. Fernández Quijada y M. Ramos-Serrano (Eds.), Tecnologías de la persuasión: uso de las TIC en publicidad y relaciones públicas. (pp. 181-196). UOC.

Singh, S. \& Sonnenburg, S. (2012). Brand performances in social media. Journal of Interactive Marketing, 26(4), 189-197. https://doi. org/10.1016/j.intmar.2012.04.001

Statista. (2018). Distribution of Instagram users worldwide as of July 2020, by age group. https://www.statista.com/statistics/325587/instagram-global-age-group/

The Economist Intelligence Unit. (2007). Beyond loyalty: Meeting the challenge of customer engagement. http://graphics.eiu.com/files/ad pdfs/eiu_AdobeEngagementPt_I_wp.pdf

Üçok Hughes, M., Bendoni, W. K. y Pehlivan, E. (2016). Storygiving as a co-creation tool for luxury brands in the age of the internet: A 
love story by Tiffany and thousands of lovers. Journal of Product \& Brand Management, 25(4), 357-364. https://doi.org/10.1108/ JPBM-09-2015-0970

Velar Lera, M. (2019). La construcción de la marca a través del relato: el caso de las marcas de moda de lujo en Instagram (Tesis doctoral, Universidad Carlos III de Madrid). https://e-archivo.uc3m.es/handle/10016/28661

Ventura, M. (2017, mayo 3). El consumo mundial de lujo crece un 5,6 \% en 2016, hasta 860.000 millones de euros. Modaes. https: / /www. modaes.com/back-stage/el-consumo-mundial-de-lujo-crece-un56-en-2016-hasta-860000-millones-de-euros-es.html

Villena, E. (2014). La narrativa transmedia en el modelo de comunicación de las empresas de moda internacionales: un estudio de caso. Communication Papers, 3(4), 15-21. http://dx.doi.org/10.33115/udg bib/cp.v3i04.22122

Wallace, D., Walker, J., López, T. \& Jones, M. (2009). Do word of mouth and advertising messages on social networks influence the purchasing behavior of college students? Journal of Applied Business Research, 25(1), 101-109. https://doi.org/10.19030/jabr.v25i1.1052

Wimmer, R. D. y Dominick, J. R. (1996). La investigación científica de medios de comunicación social: una introducción a sus métodos. Bosch.

Wimmer, R. D. \& Dominick, J. R. (2006). Mass communication research: An introduction. (8. ${ }^{\mathrm{a}}$ ed.). Thomson Wadsworth. 